\title{
The role of DJ-1 in anti-apoptosis
}

\author{
Haigang Ren ${ }^{1,2}$, Kai Fu', Jun Fan ${ }^{1}$, Guanghui Wang ${ }^{1,2^{*}}$ \\ From 2011 International Conference on Molecular Neurodegeneration \\ Shanghai, China. 22-24 September 2011
}

\section{Background}

DJ-1 is a protein in association with Parkinson's disease (PD) and cancers. DJ-1 has been reported to exhibit both cytoplasmic and nuclear distribution (Bonifati et al., Science, 2003; Nagakubo et al., BBRC, 1997). It functions in multiple pathways to affect cell survival. It suppresses the JNK signaling pathway in cytoplasma (Mo et al., Cell Death Differ, 2008) and interacts with Daxx and sequesters it within the nucleus, preventing the initiation of apoptotic signaling (Junn et al., PNAS, 2005). In contrast to its functions in cell survival, deletions or loss of function point mutations in DJ-1 are reported to be responsible for recessive early-onset Parkinson's disease (PD) (Bonifati et al., Science, 2003). The most commonly studied PD-associated mutant, L166P, is reported to be unstable and to mislocalize to the mitochondria, leading to a loss of the cytoplasmic function of DJ-1 (Bonifati $e t$ al., Science, 2003). Although lines of evidence suggest that a high expression of DJ-1 enhances cell survival and loss of DJ-1 function is associated with PD, the detailed mechanisms are still not fully understood.

\section{Results}

In our studies, we show that DJ-1 functions in multiple ways to affect cell survival. It inhibits TRAIL-induced apoptosis by blocking Fas-associated protein death domain (FADD)-mediated pro-caspase-8 activation. Wild-type DJ1, but not the PD-associated mutant L166P, binds to FADD to inhibit the formation of the death-inducing signaling complex (DISC). DJ-1 competes with pro-caspase-8 to bind to FADD at the death effector domain (DED), thereby repressing the recruitment and activation of procaspase- 8 to the active form of caspase- 8 , suggesting that DJ-1 protects against TRAIL-induced apoptosis through the regulation of DISC formation. DJ-1 and DJ-1(L166P) have potential roles in mitochondria. DJ-1(L166P) but not DJ-1 co-localizes with and interacts weakly with $\mathrm{Bcl}-\mathrm{X}_{\mathrm{L}}$, whereas both DJ-1 and DJ-1(L166P) increase in mitochondria in response to ultraviolet $B$ (UVB) irradiation and have increased binding to $\mathrm{Bcl}-\mathrm{X}_{\mathrm{L}}$. Moreover, DJ-1 but not DJ-1(L166P) stabilizes Bcl- $\mathrm{X}_{\mathrm{L}}$ by inhibiting its ubiquitination in response to UVB irradiation. Besides its role in cytoplasma, DJ-1 exerts its cytoprotection through inhibiting nuclear p53. DJ-1 interacts with p53 in vitro and in vivo. Overexpression of DJ-1 decreases the expression of Bax and inhibits caspase activation, while knockdown of DJ-1 increases Bax protein level, and accelerates caspase-3 activation and cell death induced by UV exposure. A sumoylation-deficient mutant of DJ-1, DJ-1(K130R), shifts from nucleus to cytoplasm and fails to repress p53 transcriptional activity on Bax promoter.

\section{Conclusion}

Our data provide evidence that DJ-1 plays important roles in anti-apoptosis by its function in multiple pathways.

\section{Author details}

'Laboratory of Molecular Neuropathology, Department of Pharmacology, Soochow University College of Pharmaceutical Sciences, Suzhou, Jiangsu 201203, China. 'Laboratory of Molecular Neuropathology, Key Laboratory of Brain Function and Diseases and School of Life Sciences, University of Science \& Technology of China, Chinese Academy of Sciences. Hefei, Anhui 230027, China.

Published: 7 February 2012

doi:10.1186/1750-1326-7-S1-L16

Cite this article as: Ren et al:: The role of DJ-1 in anti-apoptosis. Molecular Neurodegeneration 2012 7(Suppl 1):L16.

* Correspondence: wghui@ustc.edu.cn

'Laboratory of Molecular Neuropathology, Department of Pharmacology, Soochow University College of Pharmaceutical Sciences, Suzhou, Jiangsu 201203, China

Full list of author information is available at the end of the article 